\title{
A specialist nurse led liaison model of care reduced unscheduled care for acute asthma in a deprived multiethnic area
}

Griffiths C, Foster G, Barnes N, et al. Specialist nurse intervention to reduce unscheduled asthma care in a deprived multiethnic area: the east London randomised controlled trial for high risk asthma (ELECTRA). BMJ 2004;328:144.

\section{Q Does a specialist nurse led liaison model of care reduce unscheduled care for patients with acute asthma in a deprived multiethnic area?}

\section{METHODS}

-

Design: cluster randomised controlled trial.

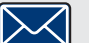

Allocation: concealed.

Blinding: blinded (outcome assessors and data analysts).

Follow up period: 1 year.

Setting: 44 general practices in east London, UK.

Patients: 324 patients who were 4-60 years of age (mean age 23 y, 50\% men, $51 \%$ South Asian), had physician diagnosed asthma, and were admitted to or attended hospital or general practitioner after-hours services with acute asthma.

$\mathbf{R}_{\mathbf{X}}$

Intervention: general practices were stratified by partnership size, training practice status, hospital admission rate for asthma, and employment of a practice nurse and whether he or she was trained in asthma care. 22 practices (175 patients) were allocated to a liaison model of care: two $1 \mathrm{~h}$ asthma specialist nurse visits to each practice to discuss acute asthma management guidelines; specialist nurse review of patient asthma control and drugs (identification of high risk asthma, assessment of inhaler technique and peak expiratory flow, and self management advice); liaison with general practitioners and practice nurses; and ongoing clinical support. 22 practices (149 patients) were allocated to usual care and a single nurse visit to the practice to discuss standard asthma guidelines and check inhaler technique.

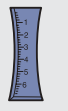

Outcomes: attendance and time to first attendance for unscheduled asthma care (consultation in primary, secondary, or after-hours care with signs and symptoms of acute asthma). Secondary outcomes included self management behaviour (increased use of bronchodilators, inhaled corticosteroids, or peak flow meter) and quality of life.

Patient follow up: $98 \%$ (intention to treat analysis).

\section{MAIN RESULTS}

Fewer patients in the intervention group than the usual care group had unscheduled asthma care at 1 year (table). The intervention delayed time to first attendance for unscheduled asthma care (median $194 v 126 \mathrm{~d}$, adjusted hazard ratio for time to first unscheduled attendance $0.73,95 \%$ CI 0.54 to 1.00 , borderline significance), but did not affect self management behaviour.

\section{CONCLUSION}

A specialist nurse led intervention reduced unscheduled care for acute asthma in a deprived multiethnic area.

For correspondence: Professor C Griffiths, University of London, London, UK. c.j.griffiths@qmul.ac.uk

Source of funding: National Asthma Campaign.
Specialist nurse led liaison model of care $(\mathrm{LMC}) \mathrm{v}$ usual care (UC) for acute asthma*

\begin{tabular}{lllll}
\hline Outcome at 1 year & LMC & UC & RRR $(95 \% \mathrm{CI})$ & NNT (CI) \\
\hline Unscheduled asthma care & $58 \%$ & $68 \%$ & $17 \%(0.3$ to 34$)$ & $9(5$ to 459$)$
\end{tabular}

*Abbreviations defined in glossary; RRR, NNT, and Cl calculated based on adjusted odds ratio and control event rate reported in article.

\section{Commentary}

7 here is increasing recognition that minority ethnic groups have higher rates of disease exacerbation and hospital admissions for asthma. ' Studies have shown that about $15 \%$ of patients discharged from hospital or emergency facilities are readmitted within 2 weeks. ${ }^{2}$ Research is limited on specific interventions that reduce unscheduled asthma care, although post-discharge asthma education involving inhaler technique, peak flow recordings, or written symptom based action plans have been shown to reduce morbidity and relapse rates. ${ }^{3}$

The studies by Castro et al and Griffiths et al assessed the effect of specialist nurse interventions on unscheduled care or hospital emergency admission rates for [mostly] minority group patients with asthma. The studies varied in methodology, but both were prospective, included patients with physician diagnosed asthma who attended the ED, and assessed health care utilisation outcomes and quality of life at 1 year. However, the study populations differed: Castro et al enrolled mainly African-American women 18-65 years of age, whereas Griffiths et al enrolled mostly South Asian, black African, or Afro-Caribbean patients aged 4-60 years. As well, in the US based study by Castro et al, the intervention occurred primarily in hospital, whereas in the UK based study by Griffiths et al, the intervention occurred primarily in general practices and comprised both patient and clinician education and support.

The lack of an economic analysis by Griffiths et al may be important because the study by Castro et al required more intensive intervention. Griffiths et al did not provide the more costly face to face nurse time or telephone contact with patients. Although both studies reduced hospital admissions, only Castro et al found differences in quality of life measures and medication use.

The findings of these 2 studies are relevant to specialist nurses working with asthma patients in primary and secondary care settings. Both studies provide support for the use of specialist nurse interventions to reduce the use of emergency facilities for asthma care and to identify the patients who will use them. The results highlight the increased use of emergency services by minority groups for asthma care. This may indicate that current service provision disadvantages these patients, and their dissatisfaction with current services may require alternative approaches to asthma care. In particular, although the study by Griffiths et al was not powered to measure differences in outcome for different ethnic groups, there was a suggestion that the intervention might provide greater benefits to white patients. Further research is required to determine the varying needs of different ethnic groups in asthma management. Both studies found that identifying patients with repeated use of emergency services and focusing interventions on these patients were beneficial. However, the precise components of the intervention and the patient groups to which they should be targeted are still to be determined. Jane E Scullion RGN, MSc Aberdeen University, Aberdeen, UK 1 Burr ML, Verrall C, Kaur B. Respir Med 1997;91:603-8.

2 Emerman CL, Woodruff PG, Cydulka RK, et al. Chest 1999;115:919-27. 3 Cowie RL, Revitt SG, Underwood MF, et al. Chest 1997;112:1534-8. 